\title{
Periodontitis and dental implant loss
}

\author{
Abstracted from
}

Sgolastra F, Petrucci A, Severino M, Gatto R, Monaco A.

Periodontitis, implant loss and peri-implantitis. A meta-analysis. Clin Oral Implants Res 2013; doi: 10.1111/clr.12319. [Epub ahead of print] PubMed PMID: 24382358.Address for correspondence: Dr Fabrizio Sgolastra, School of Dentistry, Dental Clinic, University of L'Aquila, V. le Vetoio 1, Building Delta 667100 L'Aquila, Italy. E-mail: fabrizio.sgolastra@gmail.com

\section{Question: Is periodontitis a risk factor for dental implant loss?}

Data sources Medline, the Cochrane Central Register of Controlled Trials (CENTRAL), Cochrane Database of Systematic Reviews, Database of Abstracts of Review of Effect, Science Direct, and SCOPUS databases were searched. A manual search was performed of issues of the last 15 years of the Journal of Periodontology, International Journal of Periodontics and Restorative Dentistry, Journal of Clinical Periodontology, Journal of Dental Research, Journal of Periodontal Research, Journal of Dentistry, Clinical Oral Investigations, Clinical Oral Implant Research and Clinical Implant Dentistry and Related Research. Study selection Prospective cohort studies that compared patients with periodontal disease (PD) to periodontally healthy patients and that reported data on implant loss, peri-implant bone changes or incidence of peri-implantitis were included. Case series, reviews, case reports and retrospective studies were not included.

Data extraction and synthesis Study selection and risk of bias assessment using the Newcastle-Ottawa Scale was undertaken independently by two reviewers. Dichotomous data were expressed as risk ratios (RRs) and 95\% confidence intervals (Cls). Continuous data were expressed as standardised mean differences (SMDs) and randomeffects meta-analysis conducted.

Results Fourteen studies were included with sample sizes ranging from 10-717. Meta-analysis (11 studies) showed a higher and significant risk for implant loss in patients affected by PD (RR: 1.89, 95\% Cl:

$1.35-2.66, \mathrm{P}=0.0002)$ with no evidence of heterogeneity ( $\mathrm{V} 2=4.31, \mathrm{P}$ $=0.93 ; 12=0 \%$ ). Significant implant bone loss was present in patients with PD compared with periodontally healthy patients (SMD: 0.44, 95\% Cl: 0.19-0.69, P = 0.0006) (three studies), with no evidence of heterogeneity $(\mathrm{v} 2=2.463, \mathrm{P}=0.27 ; 12=24 \%)$.

Periodontally compromised patients also showed an increased risk of peri-implantitis compared with patients without PD (RR: $2.21,95 \%$ $\mathrm{Cl}: 1.42-3.43, \mathrm{P}=0.0004)$, with moderate but not significant heterogeneity ( $\mathrm{v} 2=7.35, \mathrm{P}=0.12 ; 12=46 \%$ ). Meta-analysis (seven studies) found that patients with both aggressive and chronic periodontitis had an increased risk of implant loss. The risk was greater for patients with aggressive periodontitis (RR: $4.04,95 \% \mathrm{Cl}: 1.81-8.98, \mathrm{P}=0.0006$ ) compared with patients with chronic periodontitis (RR: $1.59,95 \% \mathrm{Cl}$ : $1.10-2.32, \mathrm{P}=0.01$ ).

When stratifying only those patients with chronic periodontitis, those with severe periodontitis had increased risks of implant loss (RR: 1.89,
$95 \% \mathrm{Cl}: 1.16-3.07, \mathrm{P}=0.01)$. Higher risks were also seen for patients with moderate (RR: $2.54,95 \% \mathrm{Cl}: 0.65-9.93, \mathrm{P}=0.18$ ) and severe periodontitis (RR: $3.12,95 \% \mathrm{Cl}$ : 0.92-10.57, $\mathrm{P}=0.07$ ).

Conclusions Strong evidence suggests that periodontitis is a risk factor for implant loss; moderate evidence revealed that periodontitis is a risk factor for peri-implantitis and that patients with periodontitis have higher implant-bone loss.

\section{Commentary}

The aim of this well organised systematic review and meta-analysis is to find scientific evidence regarding the 1) implant loss, 2) bone loss around implants and 3) peri-implantitis incidence among patients with a history of periodontal disease (PD) compared with patients without PD.

The topic has been discussed since the placement of implants in patients who lost their teeth due to PD, and many reviews are available. However, this systematic review dealt with prospective studies only, which makes this article more noteworthy. The materials section adequately addressed how the systematic review was conducted.

They followed the recommendations of the Cochrane Collaboration and the PRISMA statement, and if you apply other measurement tools, such as AMSTAR, ${ }^{1}$ we would find that this systematic review put a lot of effort into establishing proper methods.

Meta-analysis showed that higher and significant amounts of 1) implant loss, 2) bone loss around implants and 3) risk of periimplantitis incidence were present in patients with PD (Fig. 2). Subgroup analysis was only possible for implant loss. When analysis was performed on chronic periodontitis and aggressive periodontitis patients, both groups showed increased risk of implant loss. However, when comparing moderate and severe periodontitis with healthy groups, no significantly increased risks were found (Fig. 3).

Heterogeneity analysis revealed that some of the groups compared showed increased heterogeneity index (risk of implant bone loss in periodontitis vs healthy groups, $\mathrm{I} 2=24 \% /$ risk of peri-implantitis in periodontitis vs healthy groups, $\mathrm{I} 2=46 \%$ ). However it was not considered significant. Moreover, a random effect model was applied, expecting inter-study heterogeneity.

Several important heterogeneities were discussed in the discus- 
sion section. Among those, time of follow-up and discrepancy of baseline difference should be borne in the reader's mind.

When setting the baseline, especially for the implant bone loss, one study set the baseline as one year after implant surgery, ${ }^{2}$ while the others set the baseline as delivery of suprastructure..$^{3,4}$ The bone loss around an implant is most dynamic during the first year after surgery, so this discrepancy might make some differences among the pooled samples.

Also, the degree of periodontitis treatment was not possible to match among the studies. Although all the patients were reported to receive periodontal therapy, only two of the studies in the systematic review reported that treatment was successful, and the others did not report any results from the treatment.

This may be an important issue in terms of the incidence of periimplantitis, as numbers of untreated residual pockets were proposed to be a risk indicator for peri-implantitis. ${ }^{5}$

Dong Won Lee

Department of Applied Oral Sciences, The Forsyth Institute, Cambridge, MA, USA

\section{Practice points}

- Patients with a history of periodontitis are prone to increased implant loss, implant bone loss and peri-implantitis.

- The risks are higher with aggressive periodontitis.

- Even though all of the patients in the periodontitis groups underwent periodontal treatment, inferior results occurred. Thus, clinicians need to pay extra caution with patients with periodontal disease, before and after performing implant surgery.

1. Shea B], Grimshaw JM, Wells GA, et al. Development of AMSTAR: a measurement tool to assess the methodological quality of systematic reviews. BMC Med Res Methodol 2007; 7: 10.

2. Karoussis IK, Salvi GE, Heitz-Mayfield LJA, Brägger U, Hämmerle CHF, Lang NP. Longterm implant prognosis in patients with and without a history of chronic periodontitis: a 10-year prospective cohort study of the ITI Dental Implant System. Clin Oral Implants Res 2003; 14: 329-339.

3. Mengel R, Flores-de-Jacoby L. Implants in patients treated for generalized aggressive and chronic periodontitis: a 3-year prospective longitudinal study. J Periodontol 2005; 76: $534-543$

4. Roccuzzo M, De Angelis N, Bonino L, Aglietta M. Ten-year results of a three-arm prospective cohort study on implants in periodontally compromised patients. Part 1: implant loss and radiographic bone loss. Clin Oral Implants Res 2010; 21: 490-496.

5. Cho-Yan Lee J, Mattheos N, Nixon KC, Ivanovski S. Residual periodontal pockets are a risk indicator for peri-implantitis in patients treated for periodontitis. Clin Oral Implants Res 2012; 23: 325-333.

Evidence-Based Dentistry (2014), 59-60. DOI: 10.1038/sj.ebd.6401031 